\title{
Commissioning of the ATLAS Level-1 Central Trigger System
}

\author{
Daniel Sherman*,a for the ATLAS Collaboration ${ }^{1}$ \\ ${ }^{a}$ CERN, CH-1211 Geneva 23, Switzerland
}

\begin{abstract}
The ATLAS level-1 central trigger is a critical part of ATLAS operation. It receives the $40 \mathrm{MHz}$ bunch clock from the LHC and distributes it to all sub-detectors. It initiates their read-out by forming the Level-1 Accept decision, which is based on information from the calorimeter and muon trigger processors and a variety of additional trigger inputs from detectors in the forward region. It also provides trigger summary information to the data acquisition system and the Level-2 trigger system. In this paper, we present the completion of the installed central trigger system, its performance during cosmic-ray data taking and the experience gained with triggering on the first LHC beams.
\end{abstract}

Key words:

Trigger systems, Large Hadron Collider, Cosmic ray commissioning

\section{Introduction}

The successful commissioning of the ATLAS Level-1 trigger, which includes components of the calorimeter and muon systems, is a critical step in preparations for $\mathrm{LHC}$ collisions. The Level-1 Central Trigger (L1CT), composed of the Central Trigger Processor (CTP), Muon-to-CTP Interface (MuCTPI), and multiple Timing, Trigger and Control (TTC) partitions, serves as the primary interface between the experiment and the accelerator, and additionally is responsible for the global timing and synchronization of all ATLAS sub-detectors. The L1CT system operates at the bunch crossing frequency of the Large Hadron Collider (LHC), approximately $40 \mathrm{MHz}$, and distributes all Level-1 Accept decisions (L1A) to all ATLAS sub-systems.

A block diagram of the Level-1 trigger is shown in Figure 1. The system has been complete and in use since the end of 2007, including the period in September 2008 with the first LHC beams. In this document, we describe the architecture of the L1CT system, and we present results from two phases of ATLAS commissioning, with cosmic ray data and with single LHC beams; this includes the development of two specific trigger sources, the Minimum Bias Trigger Scintillators (MBTS) and Beam Pickups (BPTX), which are introduced in Section 4.

\section{Functions of the Central Trigger}

The central trigger receives trigger information from the calorimeter and muon systems, the latter through the MuCTPI. The information is in the form of multiplicities

\footnotetext{
*Corresponding author

Email address: daniel.sherman@cern.ch (Daniel Sherman)
}

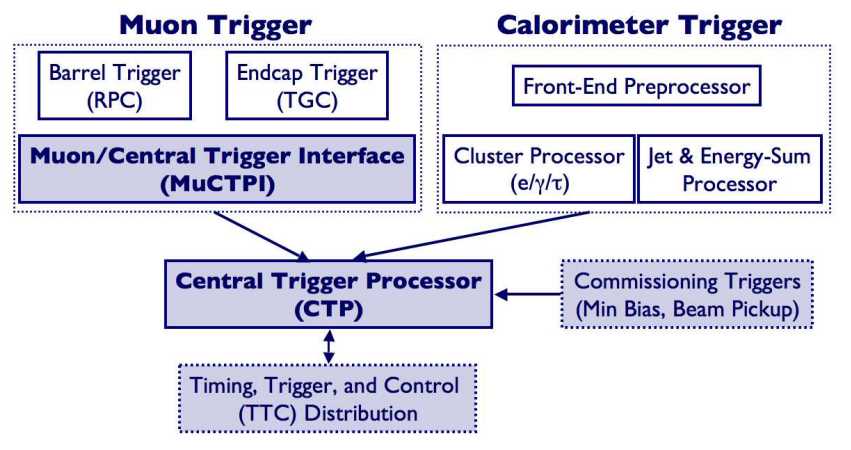

Figure 1: Block diagram of the ATLAS Level-1 trigger system. Blocks that are shaded are the components of the L1CT. The central trigger receives information from the calorimeter and muon systems (through the MuCTPI) and passes the trigger decision back to each sub-system via its TTC Partition. The Level-1 trigger also includes minimum bias scintillators and beam pickups.

of reconstructed objects (electrons, photons, jets, and taus from the calorimeter, muon track fragments from the muon systems), which are evaluated for several transverse energy thresholds, as well as a global calculation of the total transverse energy and the missing transverse energy in the event. The CTP also accepts direct trigger inputs, such as those from the MBTS and BPTX, and internal triggers, such as random triggers, prescaled clocks, or fixed bunch crossing numbers. [1]

The CTP internally synchronizes and aligns the trigger inputs to have the full event contents. Based on these data, it evaluates a L1A decision according to a programmable trigger menu, consisting of 256 logical combinations of the raw CTP inputs. [1] For example, the trigger menu may have items including both electrons and muons, though 
the calorimeter and muon trigger information is separate before reaching the CTP. The L1A is the logical OR of all trigger items in the menu.

Finally, the CTP distributes the trigger decision to all the sub-systems through their TTC partitions. In the case of an accepted event, the sub-systems pass the event information on to the Level-2 trigger for slower, more advanced reconstruction. The CTP also passes the Region of Interest, the physical location of the triggered objects in the detector, to Level-2 to seed its reconstruction.

\section{Components of the L1CT System}

\subsection{Central Trigger Processor}

The CTP consists of five distinct types of boards housed in a $9 \mathrm{U}$ VME crate. The CTP core module (CTP_CORE) receives the trigger inputs, handles the internal trigger sources, and combines this information into the L1A. The trigger menu is stored in the CTP_CORE, as is a prescale set for controlling the trigger rates for each item. [2]

The CTP input module (CTP_IN) receives trigger inputs directly from the trigger processors, aligns them with each other, and synchronizes them with the internal clock. The output module, the CTP_OUT, passes timing information and the L1A from the CTP out to the ATLAS sub-detectors. It may also receive requests from the subdetectors, such as a busy signal or a request for a calibration event, which it passes back to the CTP_CORE. There are 3 CTP_IN and 4 CTP_OUT modules in the CTP crate.

The CTP machine interface (CTP_MI) board receives timing signals from the LHC and provides the overall timing reference for ATLAS. Finally, the CTP monitoring module (CTP_MON) records trigger counts and rates for each item and bunch crossing number, which may used to monitor the beam structure and quality. [2]

\subsection{Muon-to-CTP Interface}

Where the calorimeter trigger has a dedicated processor for determining energies and multiplicities outside the central trigger, the muon trigger system is composed of two separate technologies, thin-gap chambers (TGC) and resistive plate chambers (RPC), which report separately to an intermediate processor, the MuCTPI. [1] The MuCTPI is responsible for aligning and counting muon candidates from each sub-detector, reporting only the total multiplicities to the CTP. Like the CTP, the MuCTPI is composed of several different boards housed in a $9 \mathrm{U}$ VME crate.

The MuCTPI primarily consists of sixteen identical modules, the octant boards (MIOCT's), each receiving muon candidate information from a $45^{\circ}$ slice of one end of the detector. These candidates are aligned in time and passed to a special backplane (MIBAK), which sums the candidates from each MIOCT. These total multiplicites, for six different $p_{\mathrm{T}}$ thresholds, are sent to the CTP through a third module, the MICTP, which also receives timing signals and the L1A from the CTP. If the event is accepted, the MuCTPI readout driver (MIROD) sends an event summary, including Regions of Interest, to the Level-2 trigger and the data acquisition system. [3]

\subsection{Timing, Trigger, and Control}

The CTP communicates with each ATLAS sub-detector through a common system, called a Timing, Trigger, and Control partition. An important piece of hardware in such a partition is the Local Trigger Processor (LTP), which is capable of receiving signals from the CTP, such as bunch counters, bunch and event counter resets, and L1A's, or, alternatively, of generating such signals in the case of a standalone run. [4] It is this functionality of the LTP that allows the sub-systems to take data independent of the rest of ATLAS, in particular without requiring the use of the CTP.

Further, many partitions are equipped with an LTP interface board (LTPi) between the CTP and LTP. These LTPi's may be linked to one another and operated as multiple slaves with one master, so that groups of sub-systems with multiple LTP's may operate together without the use of the CTP; for instance, the full ATLAS muon system uses six different TTC partitions, but through the LTPi's they may collectively run independent of the central trigger.

\section{Commissioning}

Since the L1CT system has been operational, the commissioning of the ATLAS detector has gone through two distinct phases, with cosmic ray events and with single LHC beams. During cosmic ray commissioning, the full readout system and analysis chain can be tested, though the physics content, event rate, and running conditions are unlike those expected during operation of the LHC; the single-beam commissioning period, in September 2008, was much more useful for studying the L1CT performance with the LHC on, though the data-taking period was limited to just a few days. In this section, we discuss L1CT activities during these two phases.

\subsection{Commissioning Triggers}

Since the CTP can accept direct trigger inputs from external sources, we have developed two sets of triggers specifically designed to assist with validation and timing studies for L1CT. The first of these, the Minimum Bias Trigger Scintillators (MBTS), have been used during both the cosmic ray and single-beam periods. The system consists of two wheels of scintillator panels, each mounted face-on to the beam axis 3.5 meters on either side of the interaction point. The 32 individual panels provide independent triggers, giving a clear indication of when and where a track passes parallel to the beam axis.

The beam pickup system, or BPTX, consists of two sets of four electro-static pickups, mounted on the beampipe 175 meters upstream of the ATLAS interaction point. The BPTX are sensitive to the passage of each beam, and 
therefore provide to the CTP a stable time reference between the LHC and L1CT. This trigger was used only during the single-beam period, but was critical for distinguishing real beam triggers from cosmic rays or other triggers and determining the true relative timing of the various triggers for collision data.

\subsection{Cosmic Ray Commissioning}

The ATLAS detector has been acquiring data since the end of 2007, operating a full system from early 2008. Over the course of this time, the detector has acquired more than 300 million events from cosmic ray muons. The data were acquired with L1A rates up to a few hundred events per second, and the vast majority of events only triggered a single menu item. Roughly $80 \%$ of these were triggered by the RPC system, which covers the top and bottom of ATLAS and is the primary target for downward-traveling muons.

Both the CTP and MuCTPI operated smoothly during this period, though the data was of limited utility to study the true performance of the L1CT. In events with only one trigger item fired, it is impossible to understand the real timing of the trigger, nor is it possible to study the true trigger efficiency without knowing a priori the rate of cosmic ray events in the detector. Further, since cosmic rays can arrive at any time within the bunch crossing window, and the event geometry can vary greatly, the timing resolution from events with multiple triggers could only be determined with a precision of roughly 1 bunch crossing, or $25 \mathrm{~ns}$.

\subsection{Single-Beam Commissioning}

In the first days of operation of the LHC, both the clockwise and counter-clockwise beams were tested, one at a time. In each case, the runs started with several bursts of particles intentionally fired onto beam collimators upstream of the detector. In these events, the CTP saw triggers from the BPTX, MBTS, muon, and calorimeter systems, providing an excellent sample of overlapping triggers to test the performance and timing alignment in the CTP. After these collimator shots, the beam was circulated multiple times, as the trigger alignment was iteratively corrected to match the expected timing for collisions. With only a few runs in these first days, the timing resolution for multiple sub-detectors was reduced from 3 bunch crossings, or $75 \mathrm{~ns}$, to 1 bunch crossing or less. This is demonstrated in Figure 2. The L1CT system performed well during these days, providing much-needed operational experience with beam and in dealing with overlapping trigger items.

\section{Conclusions}

The L1CT system, including the CTP, MuCTPI, and TTC partitions, has been successfully installed and operated in the ATLAS cavern. Through the cosmic ray and
Relative Trigger Timing, 10 September

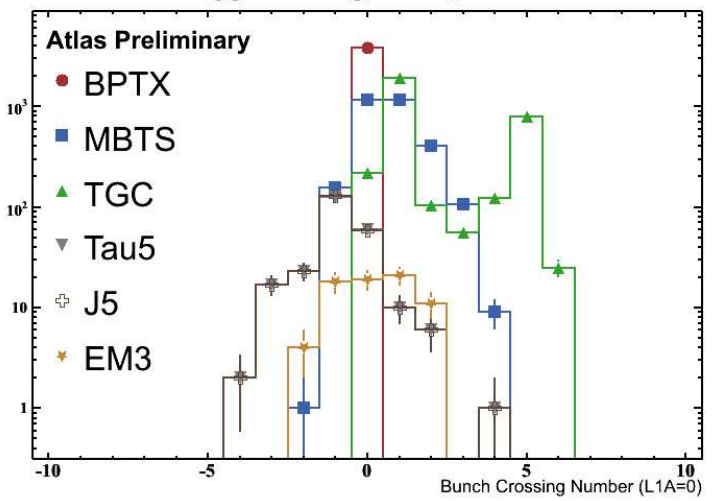

Relative Trigger Timing, 12 September

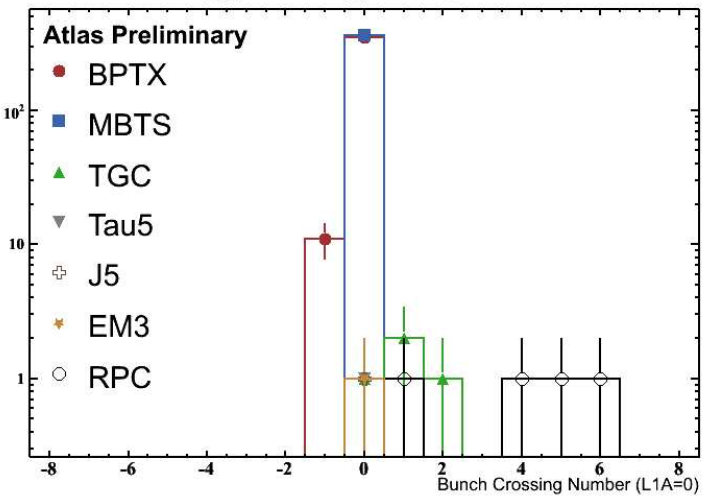

Figure 2: Trigger timing from two early single-beam runs, September 2008. The first plot shows the timing of triggers for events that triggered only on the BPTX (at bunch crossing 0). Here, the events include triggers from several other sources due to beam halo, and the timing of these other triggers is spread over several bunch crossings. The second distribution, taken two days later in a similar run, shows significantly fewer overlapping triggers as the beam quality improved, and additionally much more precise relative timing. In both figures, Tau5, J5, and EM3 are single-cluster calorimeter triggers.

single-beam data acquisition periods, the L1CT system has performed stably and efficiently. Based on these first tests, L1CT is expected to be ready for first LHC collisions.

\section{References}

[1] The ATLAS Collaboration, First-level Trigger Technical Design Report, CERN/LHCC/98014, June 1998.

[2] R. Spiwoks et al., Topical Workshop on Electronics for Particle Physics, Prague, Czech Republic, 3-7 Sep., 2007. pp. 217-221.

[3] D. Berge et al., Topical Workshop on Electronics for Particle Physics, Prague, Czech Republic, 3-7 Sep., 2007. pp. 453-457.

[4] P. B. Amaral et al., The Atlas Local Trigger Processor (LTP), IEEE Trans. Nucl. Sci. 52 (2005) 1202. 